\title{
Synchronized monochromator and insertion device energy scans at SLS
}

\author{
J. Krempaský, U. Flechsig, T. Korhonen, D. Zimoch, Ch. Quitmann, F. Nolting \\ Paul Scherrer Institut, Villigen, Swiss Light Source, 5235 Villigen PSI, Switzerland
}

\begin{abstract}
Synchronous monochromator and insertion device energy scans were implemented at the Surfaces/Interfaces:Microscopy (SIM) beamline in order to provide the users fast X-ray magnetic dichroism studies (XMCD). A simple software control scheme is proposed based on a fast monochromator run-time energy readback which quickly updates the insertion device requested energy during an on-the-fly X-ray absorption scan (XAS). In this scheme the Plain Grating Monochromator (PGM) motion control, being much slower compared with the insertion device (APPLE-II type undulator), acts as a "master" controlling the undulator "slave" energy position. This master-slave software implementation exploits EPICS distributed device control over computer network and allows for a quasisynchronous motion control combined with data acquisition needed for the XAS or XMCD experiment.
\end{abstract}

Keywords: On-the-fly monochromator and insertion device absorption scans, X-ray magnetic dichroism, EPICS distributed control.

PACS: 07.85.Qe 29.30.Dn 41.85.Si

\section{INTRODUCTION}

There are a number of experiments carried out at synchrotron light sources for which synchronized monochromator and insertion device energy scans (on-the-fly scans) are required. The reason is that in some experiments, such as XAS or XMCD, a conventional point-to-point approach where the monochromator and insertion device are sequentially driven to the required photon energies, is time consuming. The limiting factor is typically the accurate positioning of the mirror and grating units inside the monochromator. Their acceleration and deceleration has to be performed gently which results in appreciable time loss. On the other hand a rigorous approach for the on-the-fly energy scans requires sophisticated multi-axis motion control in which the motion trajectories are non-linear, hence difficult to implement within motion controllers already in use at SLS. Moreover the insertion device motion control is well isolated from the PGM motion control and any modifications would be at the expense of exceeding costs and complexity. Thus the only viable solution to the problem was a software based solution in which the motion control and data are available on the computer network. This solution was corroborated by uniform instrumentation control and data acquisition in EPICS. The proposed control scheme consists of a slower device (PGM) which drives a faster device (APPLE-II type undulator) by continuously scanning the PGM energy $E(\theta, \beta, t)$ where $\theta$ and $\beta$ are absolute mirror and grating angular readouts and $t$ is the time. Besides the start energy $E 1$ and final energy $E 2$ there is also a total time of the energy scan considered as user input. The constant fixed focus ( $c f f$ ) parameter allows us to trade the SIM beamline flux and resolving power. Because the proposed motion control tips the balance between them, the effects on $c f f$ during on-the-fly XAS are briefly discussed. On data acquisition side we discuss in details signal readout and show an on-the-fly XMCD experiment example.

\section{THE ON-THE-FLY CONTROL SCHEME}

Figure 1 shows the schematic layout of the SIM beamline. The beamline optics and data acquisition is controlled with four VME-systems on which the EPICS control variables are indicated in order to show the EPICS channels involved in the XAS (or XMCD) experiment. Besides $E 1$ and $E 2$ the undulator requested energy is $E_{U}$ and "rbk"

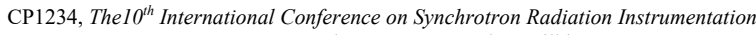
R. Garrett, I. Gentle, K. Nugent, and S. Wilkins

(C) 2010 American Institute of Physics 978-0-7354-0782-4/10/\$30.00 


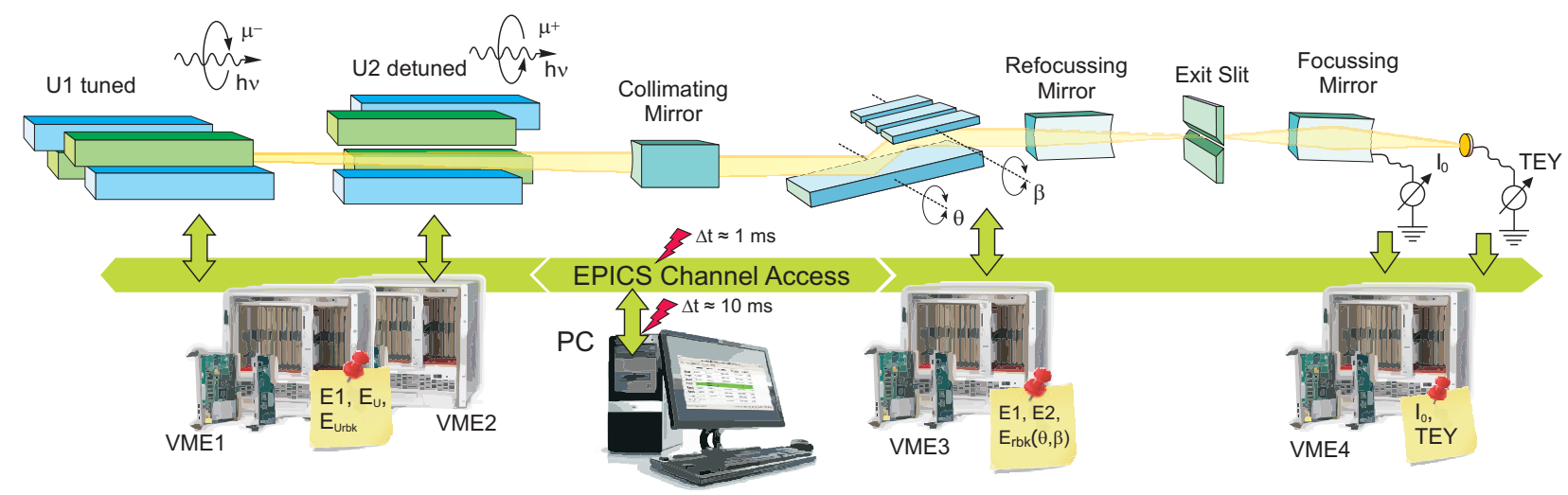

FIGURE 1. Schematic layout of the SIM beamline with underlying computer control equipment. The EPICS control variables are indicated at each of the VME crates. Also indicated are CA latencies in milliseconds (see text).

channels indicate instant PGM and undulator readback energies. On the experimental side there are two EPICS channels: the $I_{0}$ signal and the sample total electron yield current $T E Y$.

(a)

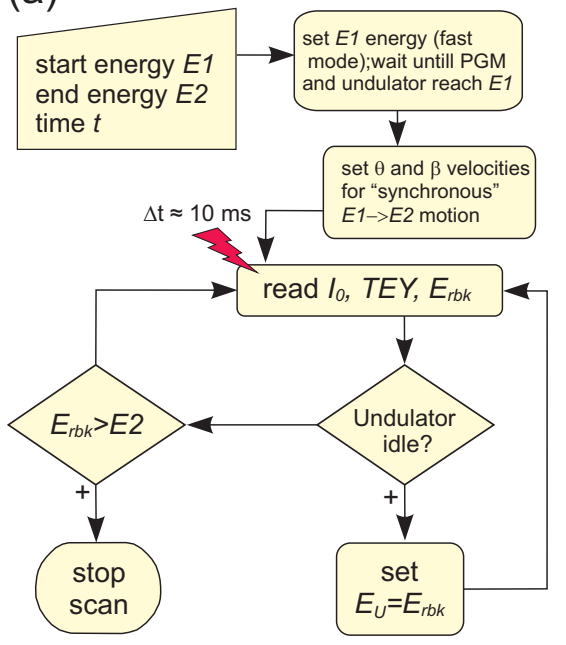

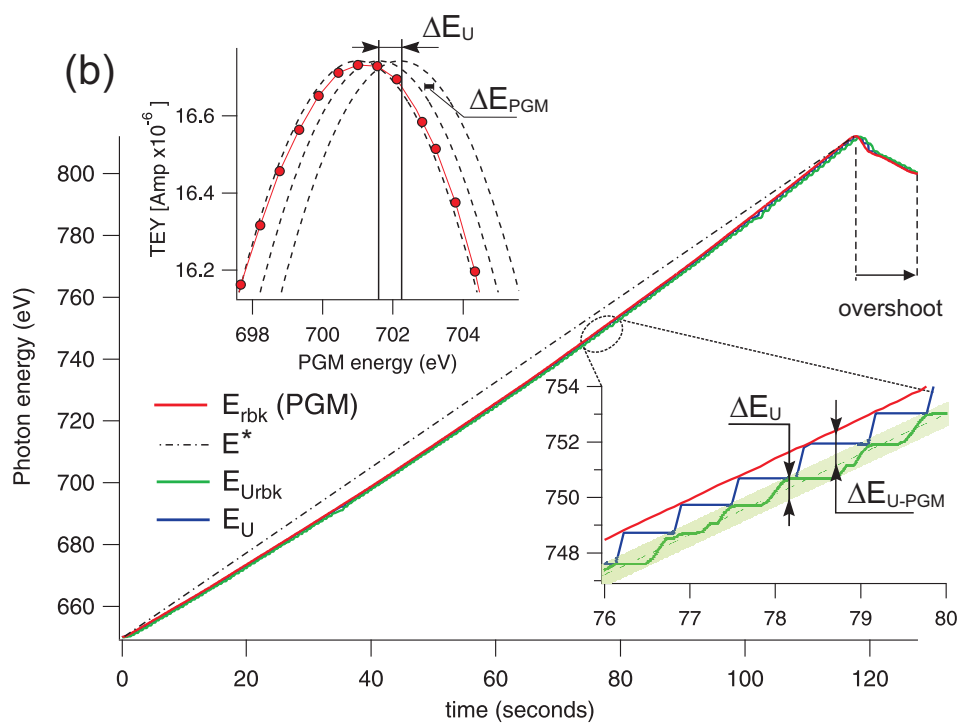

FIGURE 2. (a) Control flow of the on-the-fly application (see text). (b) On-the-fly time dependence of PGM $E_{r b k}$ and undulator $E_{U r b k}$ EPICS channels, $E_{U}$ is the undulator energy set-point, $E^{*}$ is the expected time dependency; (top in-set) Undulator energy scan around PGM fixed at $701 \mathrm{eV}$, dashed lines are Gaussian fits shifted by $\Delta E_{U}$; (bottom in-set) zoomed view on the dynamics of the on-the-fly scan (see text).

The control flow of the on-the-fly scan is shown in Fig. 2a. Once the mirror and grating velocities are optimized for a "quasi-synchronous" motion from $E 1$ to $E 2$, the key point in the control loop is to send commands to the undulator in order to align undulator energy set-point $E_{U}$ to the PGM readback energy $E_{r b k}(\theta, \beta)$. This, in turn, depends on the instant angular readouts $\theta(t)$ and $\beta(t)$. A typical XAS on-the-fly scan on oxidized Co metal sample is seen in Fig.3b, where the start energy $E 1$ is set to $760 \mathrm{eV}$ and the final energy $E 2=820 \mathrm{eV}$. Because the motion control does not allow to run non-linear trajectories, the $E_{r b k}(\theta, \beta)$ time dependency slightly drifts away from the expected energy $E^{*}$ (dot-dashed line in Fig. 2b). The side effect of this non-linearity is that the PGM $c f f_{r b k}$ varies in time as seen in Fig. 3a. However, as seen in Fig.3a', this $c f f_{r b k}$ excursion is negligible compared with the photon flux $I_{0}(c f f, E)$ distribution at the SIM beamline [1]. Another artifact introduced in the control scheme is that the $E_{r b k}(\theta, \beta, t)$ in Fig. $2 \mathrm{~b}$ has an overshoot. This is related to settling of the energy position E2 where both mirror and grating target 
positions are overshot and corrected in a closed-loop mode with external PGM encoders ${ }^{1}$. Such glitch in the motion control provides a sizeable impact even on the normalized $T E Y$ signal $\left(T E Y / I_{0}\right)$. Since the overshoot data have readback energy larger than $E 2$ they can be easily stripped off from data acquisition control loop simply by stopping the scan when $E_{r b k}>E 2$ condition occurs.

\section{RESULTS AND DISCUSSION}

Next we discuss variations of the $I_{0}$ intensity due to the overall dynamics of the undulator control. In order to illustrate the motion dynamics, the lower in-set in Fig. 2b shows PGM time dependency $E_{r b k}(\theta, \beta)$, undulator energy readback $E_{U r b k}$ and the requested undulator energy set-points $E_{U}$. There is a ripple $\Delta E_{U}$ of around $0.6 \mathrm{eV}$ in the $E_{U r b k}$ that lags behind the PGM $E_{r b k}(\theta, \beta)$ about $\Delta E_{U-P G M}=1.2 \mathrm{eV}$. Since $\Delta E_{U-P G M}$ is constant, the variations in the $I_{0}$ and $T E Y$ intensity are determined by the $\Delta E_{U}$ ripple. Because $\Delta E_{U}$ is factor 6 higher than the PGM energy resolution $\Delta E_{P G M}$, we need to estimate the effects of this ripple on the signals. Dashed lines in the top in-set of Fig. $2 \mathrm{~b}$ are Gaussian fits of the undulator energy scan at fixed PGM energy of $701 \mathrm{eV}$, shifted in energy by $\Delta E_{U}$ of $0.6 \mathrm{eV}$. The envelope of the Gaussian fits simulates intensity variations of $I_{0}$ and $T E Y$ which are of the order of $0.01 \%$ peak-topeak. The XAS signal RMS is typically $\approx 0.1 \%$ which means that in the $T E Y / I_{0}$ signal the dynamic of the on-the-fly scan can hardly affect the spectra.
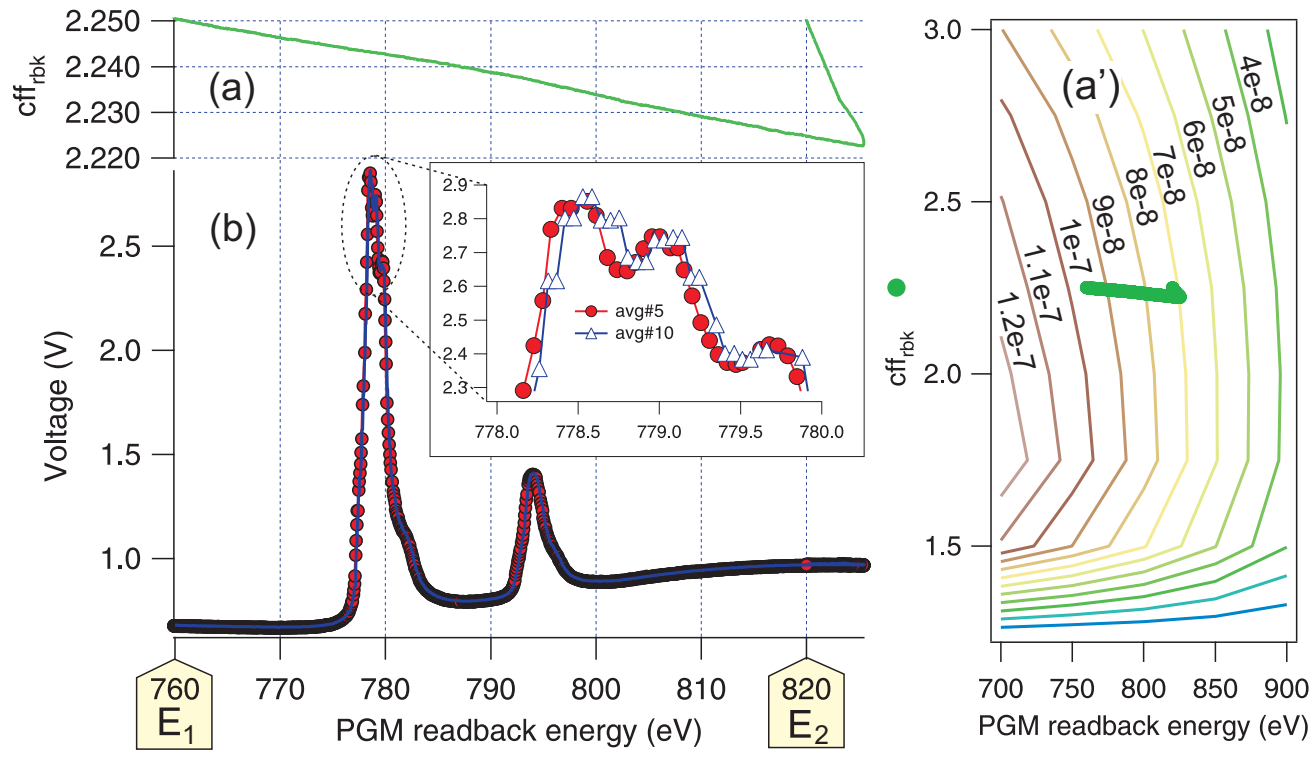

FIGURE 3. (b) $\mathrm{E} 1 \rightarrow \mathrm{E} 2$ on-the-fly XAS scan from $\mathrm{CoO}$ with zoomed view on the $\mathrm{L}_{\mathrm{III}}$ Co edge showing data with different averaging; (a) $c f f_{r b k}(E)$ excursion during the on-the-fly scan. (a') The same $c f f_{r b k}(E)$ dependency plotted on top of the $I_{0}(c f f, E)$ photon flux energy vs. cff contour plot from Ref.[1] shows that the $c f f_{r b k}$ excursion during the on-the-fly scan can hardly induce sizeable change in the photon flux (photon flux is in Amperes).

Figure $3 \mathrm{~b}$ shows on-the-fly spectra from $\mathrm{CoO}$ where $T E Y$ is normalized with $I_{0}$. $E 1$ is set to 760 and $E 2$ to $820 \mathrm{eV}$, the time of the scan is set to 2 minutes. As discussed above, the overshoot region is discarded and there remain approximately 1300 points in the spectrum - a reasonable amount of data for a state-of-the-art XMCD measurements in such a short time.

The low current signals $T E Y$ and $I_{0}$ are amplified with current amplifiers (Femto DLPCA-200 or analog output from a Keithley-type digital multimeter) and then converted into voltage via 16 bit ADC cards on a VME system. The ADC cards [2] have averaging capabilities controlled by user. There are two reasons for this: first the signal-to-noise

\footnotetext{
${ }^{1}$ In the closed-loop motion control the external encoder feedbacks the position to the motor after reaching the target position E2. This is different from motion controllers with truly active position feedback.
} 
ratio has to be reduced as much as possible; second: the EPICS channel access (CA) latency between the VME systems is around $1 \mathrm{~ms}$ and between VME and PC around $10 \mathrm{~ms}$ [3]. The latter is significant because the whole onthe-fly application is implemented on a PC which sets limits on synchronizing $T E Y / I_{0}$ data with $E_{r b k}(\theta, \beta)$. The sample averaging needs to be balanced with the CA as well. For example during a 2-minutes on-the-fly scan averaging $5 \times 1000$ samples with $10 \mathrm{kHz}$ shows reasonable data smoothing (full circles in the in-set of Fig. 3b), averaging $10 \times 1000$ samples hits the limit of the CA latency leading to under-sampled signal (triangles) because there are many adjacent $T E Y / I_{0}$ points with the same magnitude in the spectrum. Data also show overall good reproducibility in the spectra.

For the XMCD experiments a fast polarization control is essential [1]. First the PGM is set to the same energy as the center of the undulator distribution energy. For example the "U1 tuned" to $701 \mathrm{eV}$ (see Fig. 1) with left-handed circular polarization $(\mu-)$ has an energy distribution curve as seen in the top in-set of Fig. 2 with PGM being set to $701 \mathrm{eV}$. Second, the "U2-detuned" has the center shifted in energy such that there is nearly no intensity with $\mu$-polarization reaching the sample. The on-the-fly XMCD experiment thus tunes the U1 to $\mu$ - first, run the on-thefly scan, detune $\mathrm{U} 1$ and run the scan with $\mathrm{U} 2$ tuned to $\mu+$.

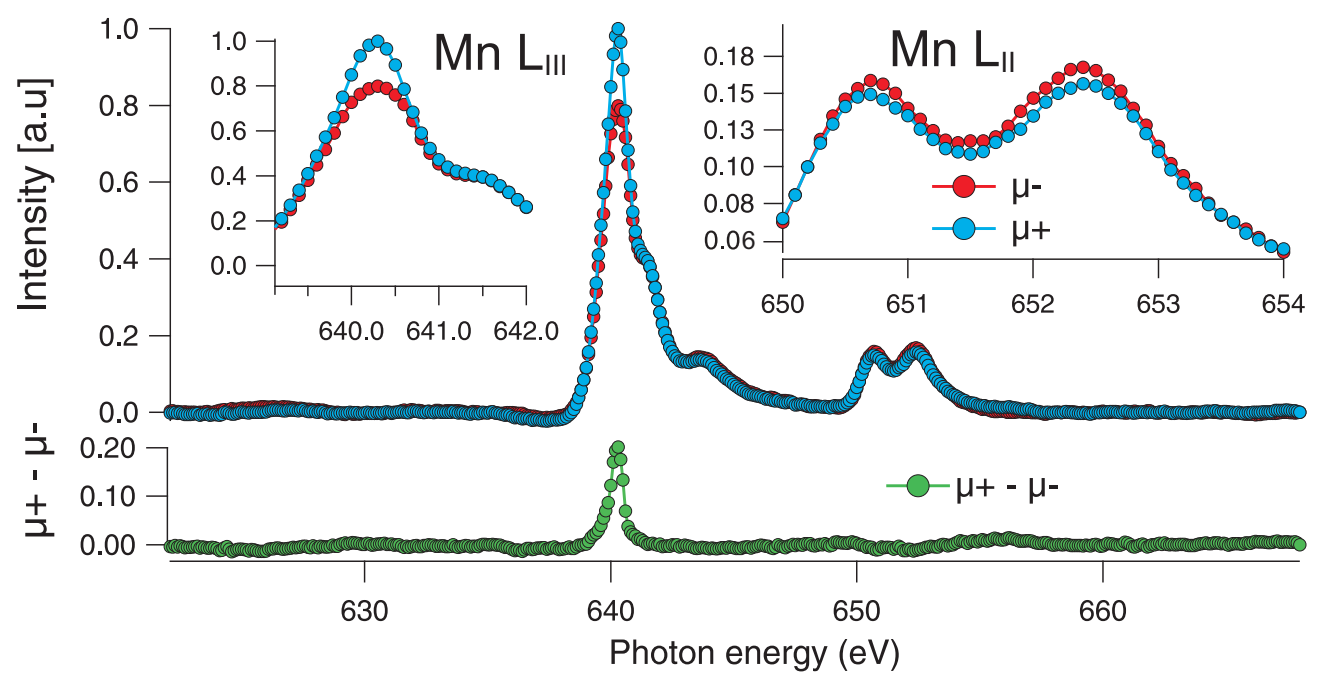

Figure 4. State-of-the-art XMCD spectrum on MnTPPCl submonolayer coverages with data zoomed to $\mathrm{MnL}_{\mathrm{III}}$ and $\mathrm{MnL}_{\mathrm{II}}$ edges. (courtesy T.Jung).

Finally, Fig. 4 shows XMCD data acquired with this technique on for MnTPPCl submonolayer coverages where circular dichroism is observed at the $\mathrm{Mn}_{\mathrm{IIIIII}}$-edges [4]. In such systems, where the magnetic material is forming molecular monolayers, rapid XMCD measurements are important to avoid sample irradiation damage. A rapid onthe-fly scan with U1 tuned with $\mu$ - (U2 detuned with $\mu+$ ) and vice versa is the method of choice for XMCD measurement at the SLS.

\section{ACKNOWLEDGMENTS}

We are grateful to Xiaoqiang Wang from the SLS controls group to deploy multi-threaded EPICS channel access features within a Python code for the on-the-fly application on a PC. We also acknowledge the technical support of Andrea Steger from the SIM beamline and useful discussions with Cinthia Piamonteze.

\section{REFERENCES}

1. U. Flechsig et. al, Performance measurements at the SLS SIM beamline, this proceedings.

2. http://www.hytec-electronics.co.uk

3. Shifu Xu, Martin R. Kraimer, "REAL-TIME PERFORMANCE MEASUREMENTS OF EPICS IOCCORE" in 10th ICALEPCS Int. Conf. on Accelerator \& Large Expt. Physics Control Systems. Geneva, 10 - 14 Oct 2005, PO2.075-5 (2005).

4. A. Scheybal, T. Ramsvik, R. Bertschinger, M. Putero, F. Nolting, T.A. Jung, Chemical Physics Letters 411 (2005) 214-220. 